\title{
DIABETES MELLITUS COMO FATOR PROGNÓSTICO EM DOENÇA CEREBROVASCULAR ISQUEMICA
}

\author{
TEREZINHA V. OLIVEIRA* - ANA MARLENE GORZ ** \\ PAULO ROGERIO M. BITTENCOURT:**
}

\begin{abstract}
RESUMO - Como parte de estudo prospectivo de 347 casos de doenca cerebrovascular isquêmica (DCVI) internados em um hospital geral, foram avaliados 36 pacientes diabéticos e 36 controles com sexo, idade e pressảo arterial semelhantes, com o objetivo de verificar como diabetes mellitus afeta o prognóstico em DCVI. Os 72 pacientes apresentavam vários tipos de DCVI (trombose, tromboembolismo ou embolia cardiogenica). Os pacientes diabéti$\cos$ tiveram estatisticamente mais dias de internamento $(p<0,05)$, mais complicações durante o internamento $(\mathrm{p}<0,05)$, mais complicaçoes infecciosas $(\mathrm{p}<0,01)$ e maior número de óbitos ao fim do seguimento $(\mathrm{p}<0,05)$. Após $377 \pm 429$ (média \pm desvio padrão) dias de seguimento, em $50 \%$ dos diabéticos havia ocorrido o óbito, enquanto após $387 \pm 405$ dias $25 \%$ dus não diabéticos haviam falecido. Os óbitos em ambos os grupos ocorreram em média no oitavo mês após o evento vascular que motivou a admissão inicial. Não foram notadas diferenças com respeito ao número de óbitos durante a internação inicial ou as condições neurológicas dos sobreviventes ao fim da admissão inicial e do seguimento.
\end{abstract}

\section{Diabetes mellitus effect on prognosis in ischemie cerebral vascular disease}

SUMMARY - As part of a prospective study about 347 cases of ischemic cerebral vascular disease (DCVI) admitted to a general hospital 36 diabetic and 36 non-diabetic patients with similar sex, age and blood pressure were evaluated with the objective of assessing the effect of diabetes mellitus on prognosis in DCVI. All patients had various types of DCVI (thrombosis, thromboembolism or cardiac embolism). The diabetic patients had statisticaly longer admissions ( $p<0.05$ ), more complications during cdmission $(\mathrm{p}<0.05)$, more infectious complications $(p<0.01)$ and a greater number of deaths at the end of follow-up ( $p<0.05)$. After $377 \pm 429$ (mean \pm standard deviation) days of follow-up, 50\% of the diabetics had died while after $387 \pm 405$ days $25 \%$ of the non-diabeties had died. Deaths in both groups occured in average at the 8th month after the vascular event that led to the first admission. There were no differences with respect to the number of deaths during admission or to neurological conditions of the survivors at the end of the first admission or at the end of follow-up.

Diabetes mellitus é amplamente reconhecido como fator predisponente de morbidade e mortalidade quando associado a doenças cerebrovasculares isquêmicas $5,12,21$, uma associação frequente na prática clínica. Estudos indicam que a ocorrência de doenças cerebrovasculares em diabéticos é 2 a 6 vezes mais frequente que em não diabéticos 11. Nos estudos de Framingham a prevalência de infartos trombóticos foi 2,5 vezes maior em homens diabéticos e 3,6 vezes maior em mulheres diabéticas que em pacientes não diabéticos 17 . Há, no entanto, poucos relatos sobre a história natural de pacientes diabéticos após um evento agudo isquêmico, dificultando avaliação objetiva do prognóstico de tais casos 1,16. Entre outros fatores de risco para doenças

Unidade de Neurologia Clínica, Hospital Nossa Senhora das Graças (HNSG), Curitiba:

* Médica Residente; ** Neurologista: *** Chefe da Unidade. 
cerebrovasculares isquêmicas destacam-se hipertensão arterial e doenças cardiacas embolígenas (endocardite bacteriana, infarto agudo de miocárdio, doença reumática, outras valvopatias e arritmias, principalmente fibrilação atrial) $\mathbf{2 , 7 , 9 , 1 0 , 1 4 , 1 9 , 2 1}$.

Neste trabalho avaliamos de maneira prospectiva e controlada a história natural de episódios agudos de doença cerebrovascular isquêmica visando quantificar objetivamente a contribuição prognóstica do diabetes mellitus e sua possivel relação com fisiopatologia e localização do processo isquêmico.

\section{MATERIAL E METODOS}

Entre 1982 e 1986 foi realizado estudo prospectivo incluindo 347 casos de doenças cerebrovasculares isquêmicas internados na Unidade de Neurologia Clínica do HNSG (20). Para o presente estudo foram determinados previamente parametros para a escolha de um grupo de pacientes diabétlcos e outro de pacientes nāo diabéticos com idade, sexo e pressão arterial semelhantes. Preenchidos estes critérios os pacientes foram retirados sucessivamente do grupo geral de 347 casos. O grupo diabético foi escolhido baseado num diagnóstico médico anterior ou pelo achado de duas ou mais glicemias de jejum acima de $140 \mathrm{mg} / \mathrm{dl}$ durante a hospitalização. Todos os pacientes admitidos ao estudo tiveram rotineiramente realizados hemograma, VHS, creatinina, glicemia, sorologia para lues, raios $\mathbf{x}$ de tórax, eletrocardiograma, tomografia axial computadorizada e quando necessário outros procedimentos diagnósticos tais como eletrocurdiografia dinâmica, eletrencefalografia, angiografia ou ecocardiografia.

A pressão arterial foi considerada normal quando níveis menores do que $160 / 90 \mathrm{mmHg}$ fossem repetidamente encontrados em pacientes abaixo de 50 anos e, nos acima de 50 anos, niveis abaixo de $160 / 95 \mathrm{mmHg}$. Os pacientes que tinham cifras tensionais acima dos niveis considerados normais ou que já estavam sob tratamento anti-hipertensivo eram considerados hipertensos. Doença cardiaca embolígena foi definida de acordo com os critérios de estudo prospectivo $(13,26)$. A avaliação do prognóstico baseou-se em número de internamentos, tempo de internação, complicações durante a internação, condição clinica no momento da alta hospitalar e ao fim do tempo de seguimento. Pela avaliação clínica no momento da alta hospitalar os pacientes foram classificados em funcionais e não funcionais, de acordo com a recuperação do déficit motor e/ou de funçōes corticais. Funcionais eram os pacientes que tiveram retorno às suas funçōes profissionais ou domésticas, tornando-se independentes; não funcionais eram aqueles que tiveram permanência de déficit motor e/ou de funções corticais tornando-se dependentes. Os resultados foram calculados estatisticamente utilizando $o$ teste $t$ de Student e o teste do qui-quadrado.

\section{RESULTADOS}

Foram estudados 36 pacientes diabéticos e 36 não diabéticos. Os pacientes diabéticos do sexo feminino (22) tiveram idade de $65 \pm 11$ anos (média \pm desvio padrāo) e os do sexo masculino (14) $61 \pm 7$ anos. Os pacientes não diabéticos do sexo feminino (22) tiveram uma idade de $65 \pm 10$ anos e os do sexo masculino (14) $61 \pm 7$ anos.

No grupo de diabéticos $29(81 \%)$ tinham diagnóstico prévio de diabetes mellitus $\mathrm{e} 7$ (19\%) foram diagnosticados durante a internação. A glicemia variou de 82 a $625 \mathrm{mg} / \mathrm{dl}$ (média $280 \mathrm{mg} / \mathrm{dl}$ ). O tempo médio de história de diabetes mellitus diagnosticado foi 6,6 $\pm 9,6$ (média \pm desvio padrão), variando de 0 a 40 anos. $O$ número e porcentagem de pacientes com diversos graus de hipertensão arterial nos grupos de diabéticos e não diabétícos estão demonstrados na tabela 1. O tipo e origem do infarto nos dois grupos estăo demonstrados na tabela 2. Foi observada diferença estatisticamente significante quanto ao maior comprometimento do sistema vertebrobasilar no grupo de pacientes diabéticos.

Os 36 pacientes diabéticos foram internados 40 vezes durante $o$ tempo de seguimento enquanto os não diabéticos foram internados 37 vezes $(p>0,05)$. O tempo da internaçăo inicial foi de $15 \pm 12$ dias (média \pm desvio padrão), variando de 3 a 53 dias no grupo de diabéticos e $10 \pm 7$ dias variando de 2 a 43 dias no grupo de não diabéticos $(\mathbf{p}<0,05)$. Em 7 dos pacientes diabéticos e 4 dos não diabéticos ocorreu o óbito durante o período de internaçāo $(p>0,05)$. Não ocorreram complicaçōes clínicas durante o internamento em 17 pacientes $(47 \%)$ no grupo de diabéticos e em $26(72 \%)$ no grupo de nåo diabéticos $(p<0,05)$. Complicaçరes infecciosas ocorreram 15 vezes no grupo de diabéticos e 5 no grupo de năo 


\begin{tabular}{|c|c|c|c|c|}
\hline Grau & $\begin{array}{l}\text { Grupo Diabético } \\
\text { Número de pacientes }\end{array}$ & $\%$ & $\begin{array}{l}\text { Grupo não Diabético } \\
\text { Número de pacientes }\end{array}$ & $\%$ \\
\hline Normal & 10 & 28 & 8 & 22 \\
\hline Leve & 17 & 47 & 20 & 56 \\
\hline Moderađa & 5 & 14 & 1 & 3 \\
\hline Severa & 4 & 11 & 7 & 19 \\
\hline
\end{tabular}

Tabela 1 - Número e porcentagem de pacientes com pressão arterial normal, hipertensão arterial leve (diastólica menor que $104 \mathrm{mmHg}$ ), moderada (menor que 120mmHg) $e$ severa (igual ou maior que $120 \mathrm{mmHg}$ ) em 36 proientes diabéticos e não diabéticos com doenca cerebrovascular aguda isquêmica.

\begin{tabular}{|c|c|c|c|c|}
\hline Infarto & \multicolumn{4}{|c|}{ Grupo } \\
\hline Tipo de Infarto & Grupo & Diabético & Grupo & não Diabetico \\
\hline Trombótico & & 16 & & 20 \\
\hline Embolico (cardiogênjeo) & & 6 & & 10 \\
\hline Tromboembólico - carótida & & 2 & & 3 \\
\hline - vertebrobasilar & & 11 & & $3 *$ \\
\hline Vasculite & & 1 & & 0 \\
\hline Origem do Infarto & Grupo & Diabeticn & Gmupo & não Diabético \\
\hline Cardiaca & & 6 & & 10 \\
\hline Artérias cerebrais & & 17 & & 20 \\
\hline Carotida & & 2 & & 3 \\
\hline Șistema vertebrobasilar & & 11 & & $3 *$ \\
\hline
\end{tabular}

Tabela 2 - Tipo e origem do infarto cerebral nos 36 pacientes diabéticos e 36 não diabéticos com doença cerebrovascular isquêmica $(* p<0,01)$.

diabéticos $(\mathrm{p}<0,01)$. Complicaçōes cardiovasculares ocorreram 4 vezes no grupo de diabéticos e 2 no grupo de nāo diabéticos $(p>0,05)$. Complicaçōes metabólicas, respectivamente em 3 e 2 ocasiões, gastrointestínais em 5 e psiquiátricas em 4 no grupo de diabéticos.

Com relação ao estado clínico no momento da alta hospitalar, 13 pacientes diabéticos estavam funcionais e 16 não funcionais; no grupo de não diabéticos 17 estavam funcionais e 15 não funcionais. Os pacientes diabéticos foram seguidos por $377 \pm 429$ dias após a alta hospitalar e os não diabéticos por $387 \pm 405$ dias (média \pm desvio padrão) $(p>0,05$ ). Ao fim do seguimento 17 diabéticos tornaram-se funcionais e 1 não funcional, enquanto 23 não diabéticos tornaram-se funcionais e 4 não funcionais. Durante 0 tempo de seguimento ocorreram 11 óbitos no grupo diabético e 5 no grupo nāo diabético. 0 número geral de óbitos passou a ser $18(50 \%)$ no grupo diabético e $9(25 \%)$ no grupo não diabético $(p<0,05)$. $O$ intervalo da alta hospitalar ao óbito durante o seguimento foi de $233 \pm 156$ dias, variando de 60 a 434 dias, no grupo diabético e $239 \pm 177$ dias, variando de 15 a 425 dias, no grupo nāo diabético $(p>0,05)$. Os pacientes foram tratados de maneira semelhante durante o internamento e o seguimento. Vinte dos pacientes diabéticos e 20 dos não diabéticos receberam anticoagulantes via endovenosa durante o internamento. Quatro diabéticos e $\mathbf{9}$ não diabéticos receberam ácido acetil salicilico. Anticoagulantes via oral foram usados cronicamente durante 0 seguimento em 7 pacientes diabéticos e em 11 nāo diabéticos, enquanto 4 diabéticos e 7 năo diabéticos receberam ácido acetil salicílico. 


\section{COMENTARIOS}

A maioria das publicaçóes registra o diabetes mellitus como fator de risco em doença cerebrovascular tanto isquêmica quanto hemorrágica $1,4,8,11,12$. A natureza prospectiva deste estudo e o fato da populaçāo diabética ter tido um grupo controle com idade, sexo e niveis tensionais semelhantes torna a interpretação dos resultados mais segura e objetiva.

A maior incidência encontrada de pacientes do sexo feminino com doença cerebrovascular isquêmica e diabetes mellitus pode refletir risco aumentado de complicações vasculares entre pacientes do sexo feminino com diabetes, já que tanto no estudo prospectivo do Hospital Nossa Senhora das Graças 20 como em outros estudos da literatura 18 a frequência de doença cerebrovascular isquêmica é um pouco maior no sexo masculino do que no feminino. No estudo populacional de Rochester 15 a frequência de doença cerebrovascular como causa de óbito em pacientes diabéticos foi pouco maior em mulheres que em homens. O achado de ocorrer o tromboembolismo vertebrobasilar com maior frequência no grupo de diabéticos foi inesperado. Uma explicaçăo fisiopatológica é difícil, mas implicaçōes clínicas claras existem devido a maior indicação de anticoagulação a longo prazo nesta localização de doença vascular. A morbidade no grupo diabético foi maior, como demonstrado pelo maior tempo de internação, maior número de complicações, maior mortalidade e maior número de óbitos ao firn do seguimento. $\dot{E}$ interessante notar, no entanto, que os pacientes que sobreviveram ao evento inicial recuperaram o déficit neurológico de modo semelhante aos não diabéticos. As complicaçōes infecciosas durante o internamento ocorreram mais frequentemente no grupo de diabéticos enquanto a frequência de complicações metabólicas e vasculares foi semelhante nos dois grupos.

Estudos experimentais de isquemia cerebral 3 mostram que a hiperglicemia aumenta a severidade do dano cerebral isquêmico. Estes resultados possivelmente possam ser relacionados a uma contribuição desfavorável em doenças cerebrovasculares 4,16. Estudo realizado para investigar a influência da hiperglicemia na evolução de doença cerebrovascular e de ataques isquêmicos transitórios pela medida da hemoglobina glicosilada permitiu concluir que a hiperglicemia geralmente precede estes eventos e habitualmente năo é reconhecida 17 . Complicaçōes sistêmicas do diabetes como a angiopatia proliferativa dos pequenos vasos cerebrais e a aterosclerose cerebral severa 6,16 influenciam o fluxo sanguineo colateral da zona peri-isquêmica e podem contribuir para resultados neurológicos mais sombrios, assim como para a observação neste estudo da maior frequência de infartos em região de alto risco como o tronco cerebral, irrigado primariamente por artérias perfurantes.

Neste estudo em metade dos pacientes diabéticos e em um quarto dos não diabéticos ocorreu o óbito durante o internamento ou no ano subsequente. Este achado indica claramente uma atitude tanto do médico quanto dos familiares com relação ao prognóstico do evento vascular. O primeiro episódio de doença cerebrovascular, sua recorrência, miocardiopatia isquêmica e complicações infecciosas, principalmente pulmonares, contribuem de maneira isolada ou associada como principais causas de morte tanto em pacientes diabéticos como em não diabéticos 1 . A falta de diferença entre os dois grupos no tocante ao tempo de seguimento demonstra que ambos receberam o mesmo tipo de atendimento ambulatorial ou institucional após o evento vascular e que os grupos são de perfil psico-social semelhante. Estes fatores, determinantes da aderência ao tratamento médico em geral, enfatizam a significação dos resultados aqui apresentados. Da mesma maneira, a análise detalhada do tratamento farmacológico empregado nos dois grupos não mostrou diferenças, novamente indicando semelhanças importantes tanto nas características do atendimento, quanto nas dos grupos, quantificadas também pelo número de pacientes que receberam anticoagulação oral, esquema terapêutico que exige pacientes com características psicosociais e geográficas especiais.

Os resultados de nosso estudo são congruentes aos dados da literatura quanto à maior morbidade e mortalidade no grupo de pacientes diabéticos. $O$ pior prognóstico neste grupo foi associado à permanência hospitalar prolongada com complicações infecciosas e áreas de isquemia cerebral em regiōes de alto risco, associadas a anormalidades metabólicas desfavoráveis. 


\section{REFERENCIAS}

1. Asplund K, Hägg E, Helmers C, Lithner F, Strand $T$, Wester PO - The naturai fístory of stroke in diabetic patients. Acta Med Scand 207:417, 1980.

2. Caplan LR, D'Cruz I, Hier BD, Reddy H, Shah S - Atrial size, atrial fibrillation and stroke. Ann Neurol 19:158, 1986.

3. Courten-Myers GM, Yamaguchi S, Wagner KR, Ting F, Myers RE - Brain injury from marked hipoxia in cats: role of hypotension and hyperglycemia. Stroke 16:1016, 1985.

4. $\operatorname{Cox} \mathrm{NH}$, Lorains JW - The prognostic value of blood glucose and glycosylated haemoglobin estimation in patients with stroke. Postgrad Med J 62:7, 1986.

5. Fuller JH, Shipley MJ, Rose G, Jarrett RJ, Keen H - Mortality from coronary heart disease and stroke in relation to degree of glycaemia: the Whitehall study. $\mathrm{Br}$ Med J $287: 867,1983$.

6. Garcia MJ ,MacNamara PM, Gordon T, Kannell WB - Morbidity and mortality in diabetics in the Framingham population. Diabetes 23:105, 1974.

7. Hart RG, Coull BM, Hart D - Early recurrent embolism associated with nonvalvular atrial fjbrillation: a retrospective study, Stroke 14:688, 1983.

8. Herman B, Leyten ACM, Van Luuk JH, Frenken CWGM, Op De Coul AAW, Schulte BPM - An evaluation of risk factors for stroke in a Dutch community. Stroke $13: 334,1982$.

9. Kagan A, Popper JS, Rhoads GG, Yano $K-$ Dietary and other risk factors for stroke in Hawaiian Japanese men. Stroke 16:390, 1985.

10. Kayan A, Popper JS, Rhoads GG - Factors related to stroke incidence in Hawaii Japanese men: the Honolulu heart study. Stroke $11: 14,1980$.

11. Kuller LH, Dorman JS, Wolf PA - Cerebrovascular disease and diabetes. In United States Department of Health and Human Services: Diabetes in America. Washingion, 1985, cap 18, pg 1.

12. Lavy S, Melamed E, Cahane E, Carmon A - Hypertension and diabetes as risk factors in stroke patients. Stroke 4:751, 1973.

13. Mohr JP, Caplan LR, Melski JW, Goldstein RJ, Duncan GW, Kistler JP, Pessin MS, Bleich HL - The Harvard cooperative stroke registry: a prospective registry. Neurology $28: 754,1978$.

14. Olivares L, Catañeda E, Grifé A, Alter $\mathbf{M}-$ Risk factors in stroke: a clinical study in Mexican patients. Stroke 4:773, 1973.

15. Palumbo PJ, Elveback LR, Chu-Pin Chu MS, Connolly DC, Kurland LT - Diabetes mellitus: incidence, prevalence, survirorship and causes of death in Rochester, Minnesota, 1945-1970. Diabetes 25:566, 1979.

16. Pulsinelli WA, Levy DE, Sigsbee B, Scherer P, Plum F - Increased damage after ischemic stroke in patients with hyperglycemia with or without established diabetes mellitus. Am J Med 74:540, 1983.

17. Riddle MC, Hart $\mathrm{J}$ - Hyperglycemia, recognized and unrecognized, as a risk factor for stroke and transient ischemic attacks. Stroke 13:356, 1982.

18. Salonen JT, Pekka Puska MPH, Jaakko Tuomilehto MA, Kava Homan MA - Relation of blood pressure, serum lipids and smoking to the risk of cerebral stroke: a longitudinal study in Eastern Finland. Stroke 13:327, 1982.

19. Sherman DG, Goldman L, Whiting RB, Jurgensen K, Kaste M, Easton JD - Tromboembolism in patients with atrial fibrillation. Arch Neurol 41:708, 1984.

20. Tso-Lin C, Bittencourt PRM, Gorz AM, Mazer S - Doença cérebro vascular isquêmica: estudo prospectivo de 347 casos num hospital geral. Tema Livre. $12^{\circ}$ Congresso Brasileiro de Neurologia, 1986.

21. Wolf PA - Risk factors for stroke (Editorial). Stroke 3:359. 1985. 